TECHNOLOGY FEATURE

Going through a phase

\title{
An array of options: proteomics gets parallel
}

\section{Arrays of DNA segments have become a standard tool in molecular biology, but arrays of proteins have been more difficult to develop. Several manufacturers now offer off-the-shelf protein arrays for a variety of uses, though, and customized arrays are becoming more common as well. With the right strategy, experimenters can use these new tools to speed their work dramatically. Alan Dove reports.}

Ten years ago, biochemists approached their work serially and painfully. They would struggle to purify a single protein, then struggle to obtain a single antibody against it, then struggle to characterize the protein's structure and function. Every protein's chemistry is different, so lessons learned in one set of experiments did not necessarily accelerate the next set.

Today, biochemists are being swept up in the 'omics' craze, which preaches the gospel of massively parallel processing. Genomics is bursting at the seams with RNA-level gene expression data from transcriptional profiling arrays, so the pressure is on protein chemists to get their assays in gear and determine what happens when those transcripts get translated.

One obvious way to do this is to use proteomic arrays—-sticking different proteins to a substrate in defined spots and testing them in parallel for particular types of interactions. But just as impregnating nine women simultaneously does not produce a baby in a month, running dozens or hundreds of biochemical assays at the same time does not necessarily accelerate protein chemistry.

Still, for many biochemical studies, protein arrays are a godsend, eliminating hours of tedium and yielding highly reproducible results. Companies have flooded into the field, offering array-based products and services for everything from cytokine profiling to subcellular localization. As with any research tool, the quality of data from a protein array depends heavily on the quality of the experiment. Investigators planning to use protein arrays need to understand the strengths and limitations of these sophisticated systems.

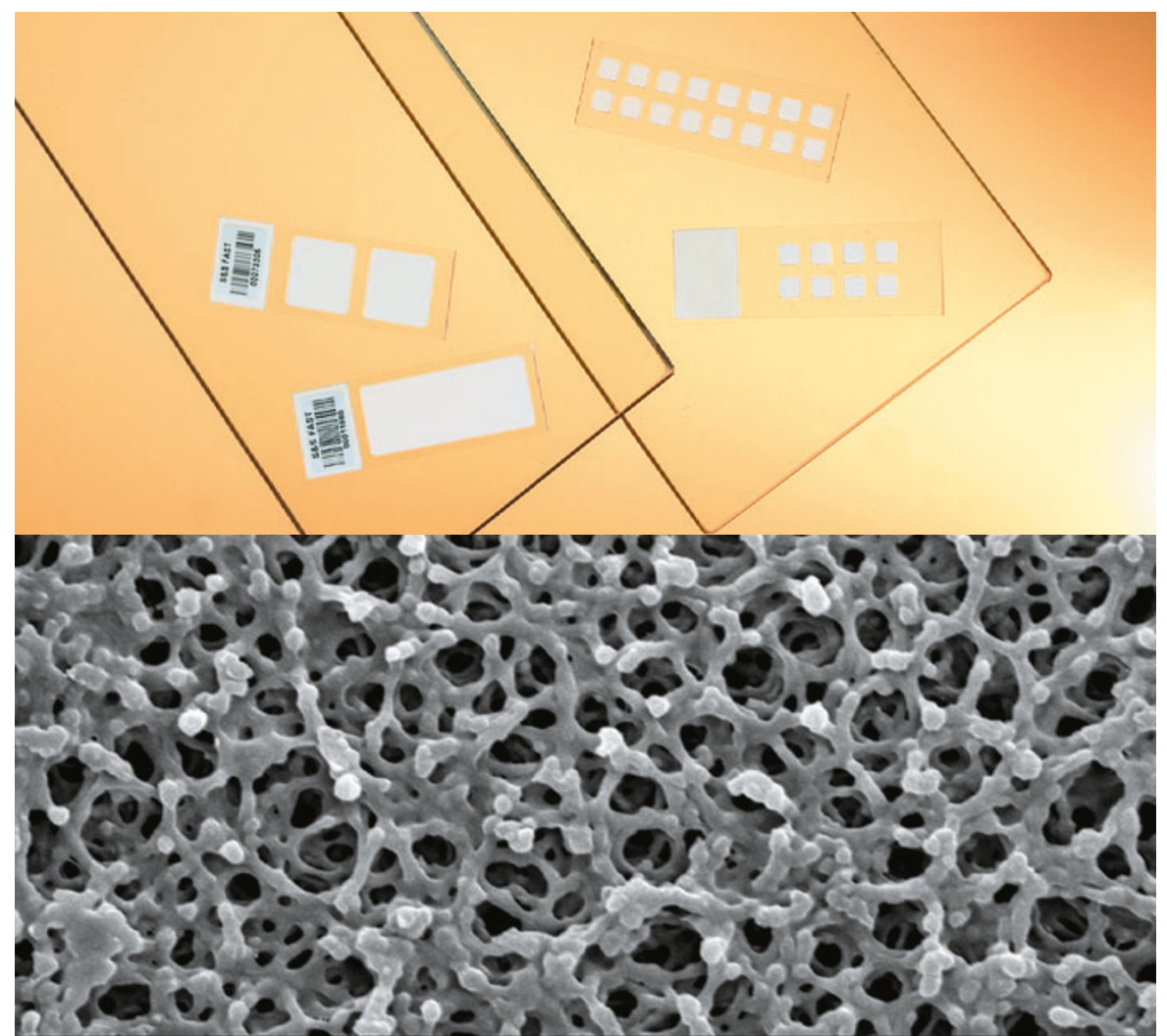

FASTslides from Whatman/Schleicher \& Schuell serve as platforms for do-it-yourself protein array construction (top). An electron micrograph of the protein-binding nitrocellulose surface of a FASTslide, ready to bind purified proteins on array spots (bottom). (Courtesy of Whatman/ Schleicher \& Schuell Bioscience.)

\section{Planting antibodies}

By far the most popular use of protein arrays is profiling the expression levels of well-known proteins. Although DNA chips can provide fast readouts of transcription, biochemists are painfully aware that posttranscriptional regulation, modification and degradation can alter actual protein levels dramatically. A definitive answer about protein levels requires a protein assay.
For researchers who only want a quick way to measure protein levels, simple arrays are available off the shelf. In these systems, each spot on the array contains antibodies against a particular target. The experimenter washes a sample over the array, then adds a labeled secondary antibody and effectively performs dozens of enzyme-linked immunosorbent assay (ELISA) procedures at once. Proteins in 
the sample bind their cognate antibodies on the array, and the higher the concentration of a protein, the brighter the spot.

“There's a substantial number of people who want to do multiplexing; it saves them time and money and sample, so we have a quantitative immunoassay system in which people can measure nine, ten or eleven different analytes," says Michael Harvey, vice president of research and development for Whatman's Schleicher and Schuell Bioscience division.

Like many other companies, Whatman sells a variety of antibody arrays in kits. Given a mouse serum sample or cell culture supernatant, measuring the levels of the dozens of circulating cytokines, key regulators of immune responses, can be as simple as following the directions in the box. Especially with quantitative assays, these mass-produced arrays often provide more reproducible results 을 than methods researchers have cobbled together themselves.

These basic antibody arrays, sometimes called analytical arrays or forward-phase
An array of arrays: each well of this 96-well plate contains 96 protein spots, for mapping the protein interactions within a specific set of signaling pathways. (Courtesy of Gavin MacBeath, Harvard University.)

arrays, are generally easy to read. Most, like the Whatman products, use fluorescent readouts that can be scanned on the same readers used for DNA arrays.

For laboratories without access to an array scanner, there are even simpler methods available. Panomics, for example, makes a cytokine profiling array with a chemiluminescent readout. "You can check this with X-ray film, or you can use an image system... that can acquire this chemiluminescence within a few minutes," says Jason Li, the company's president and CEO. Quantitation simply requires measuring the density of the resulting spots.

Because cytokine profiling is useful in so many fields, there is intense competition among manufacturers, with several offering similar products. Whatman and Panomics compete with companies like

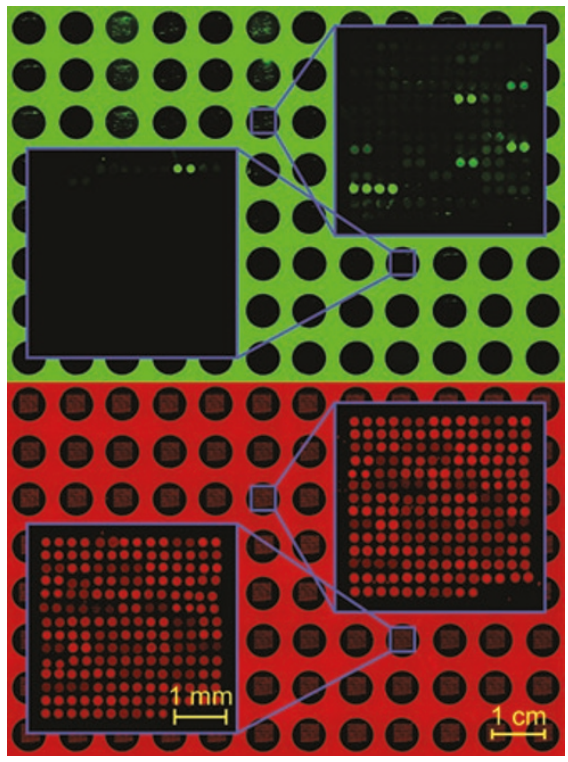

SuperArray, Zyomyx and many others in this area, so researchers should shop around before choosing a platform.

Anyone who plans to do more than a quick cytokine profiling experiment, 
though, will probably need access to some specialized equipment to read the assays. Michael Hadjisavas, global business manager for proteomics at Sigma-Aldrich, advises newcomers to "make sure you've got access to a scanner, and you've got someone who can help you with the readout and the data. Also, make sure you've got a very efficient lysis of the sample... so that you're not introducing variability."

Careful sample processing becomes especially important for those studying scarce proteins, including most intracellular signaling molecules. Detecting these proteins pushes the limits of most arrays even under ideal conditions, so dirty or inconsistent sample processing easily swamps their signals.

There are commercially available arrays for profiling intracellular signaling proteins, but researchers pursuing hard-core signal transduction research still have to blaze many of their own trails. Gavin MacBeath, assistant professor of chemistry and chemical biology at Harvard University, initially constructed his own
ELISA-style arrays for signal transduction studies, but he soon ran up against that method's limitations.

"The problem with this approach is that it's really limited by the antibodies that are currently available. It works very well for cytokines and diagnostics applications, but what we found is that... most of the antibodies that are currently available don't work well as capture reagents," says MacBeath. For signal transduction proteins, antibodies have generally been chosen for their ability to work in western blots or other denaturing assays, so they often bind poorly to intact proteins in a cell extract.

\section{Going through a phase}

To get around the limitations of the antibodies, MacBeath and many other researchers have turned to so-called reverse-phase arrays. Instead of anchoring many antibodies to the array and washing one cell extract over it, they anchor many cell extracts to the array and then probe them with one antibody at a time.
"We array lysates from cell samples onto nitrocellulose-coated slides, and then probe the slides with different antibodies," says MacBeath, who uses the approach to study tyrosine kinase signaling with phosphotyrosine and domain-specific antibodies. By arraying extracts from cells that were grown under different conditions, or different types of cells, the researchers can identify the triggers for different signals.

Although producing one's own reversephase arrays permits nearly limitless customization, it is an equipment-intensive sport (see Box 1), and many labs lack the money and time for such a project. For those who simply want to localize a favorite protein, new off-the-shelf arrays may suffice.

Sigma-Aldrich, for example, makes 'protein localization arrays', a product category that includes tissue-specific and even organelle-specific systems. "These are arrays of tissue extracts and cellular organelles, and the way these are used is to determine where a particular protein of interest is being expressed: is it in the liver,

\section{BOX 1 GEARING UP FOR ARRAYS}

Biochemists have a longstanding tradition of assembling their own equipment-indeed, the peculiar nature of protein purification and analysis often demands it. Cobbling together some columns and tubes in the cold room is a far cry from precisely measuring nanoliter volumes into near-microscopic patterns, though, and many find the prospect of making their own protein arrays daunting.

Even manufacturers selling do-it-yourself array supplies sometimes question the wisdom of doing it yourself. "I almost think it doesn't make sense for a lot of people to make their own arrays. There's so much important detail in it that it's almost worth, in most cases, having someone make it for you," says Michael Harvey, vice president of research and development at Whatman's Schleicher and Schuell Bioscience division.

Harvey's assessment isn't as self-defeating as it sounds; Whatman, like many companies in the array business, also provides custom protein array-making services. But the company still does a brisk business selling nitrocellulose-coated microscope slides and array printing devices.

As the price of automated protein arrayers has fallen into the $\$ 60,000$ range, many core facilities and some individual labs have added the capability of printing their own arrays. For those ready to take the plunge, there are two main types of machines to consider: pin-type and peizoelectric.

Pin-type arrayers are the simpler design, using tiny spikes to transfer samples onto the arrays. Automated versions are available, as are inexpensive handheld units suitable for making prototype arrays to test a concept. Pin-type systems are considered easier to use and maintain than peizoelectric arrayers, but that convenience comes at a price. "Most contact printers don't produce the same reproducibility that you get from noncontact methods," says MacBeath, a serious do-ityourself array-maker.

Instead, MacBeath relies on a peizoelectric arrayer, which drives the samples onto the plate with positive pressure, avoiding contact with the substrate. This provides greater reproducibility, but has its own quirks. "It's a really finicky technology," says MacBeath, adding that even with careful cleaning and maintenance, the system needs frequent tinkering. Peizoelectric arrayers are also more expensive than pin-type arrayers.

Once the arrays are made, either in the lab or by a manufacturer, researchers need to decide how to process and read them. Simple protein expression profiling arrays can usually be processed in an ordinary laboratory, and read on the same scanners used for DNA chips. Suitable scanners currently sell for around $\$ 40,000$.

For labs processing large numbers of arrays, especially when results need to be comparable from one array to the next, it may make sense to hire a robot. Apparatus maker PerkinElmer, for example, now offers a turnkey protein array-processing workstation for about $\$ 75,000$. That system can handle twelve arrays at once, and as many as four can be linked together to process up to 48 arrays simultaneously with different samples. 


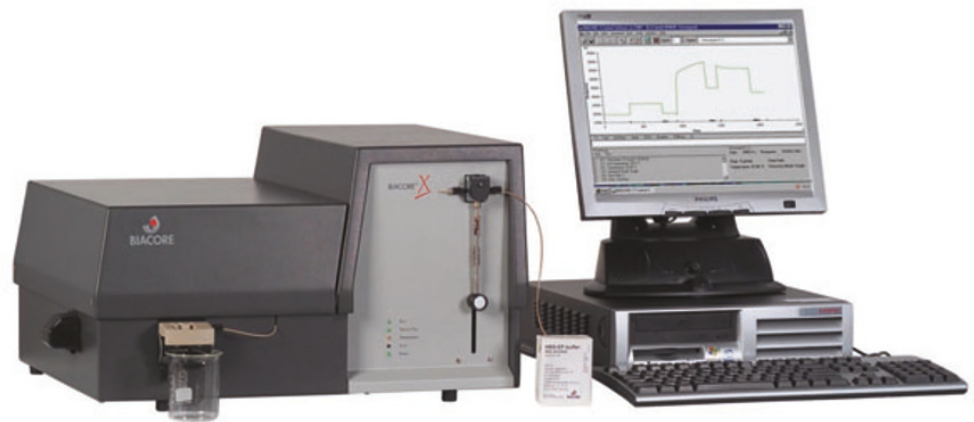

The Biacore T100 model to study protein binding on arrays. (Courtesy of Biacore, Inc.)

and if it's in the liver is it in the membrane, cytoplasm or nucleus?" says Hadjisavas.

Reverse-phase arrays can yield quick and often useful answers, but they are generally not quantitative. The intensity of a spot may indicate protein level, or it may simply indicate that a particular extract stuck to the substrate better than the dissimilar extract next to it.

Unfortunately, that point may elude researchers anxious to profile as many proteins as possible. "Different proteins like different conditions. That is a real concern... it makes me really quite suspicious of results people report when they throw down thousands of proteins, all of which are different and none of which are quality-controlled," says MacBeath.

\section{Quick, cheap and publishable}

Proteome-scale arrays may yield dirty data, but researchers willing to tolerate their limitations have found them useful nonetheless. "The human proteome is just so huge; there are all kinds of new proteins that are being identified," says Santosh Arcot, product leader for functional genomics and proteomics at PerkinElmer. Proteome-scale arrays, he says, can help organize the flood of newly discovered proteins.

One of the most popular uses for these high-throughput systems is identifying potential interacting partners for a protein of interest. Arrays are available off the shelf with thousands of semipurified proteins organized on their surfaces. Incubating these with a purified protein of interest can highlight promising candidates for more detailed analysis.

"You can actually put the entire yeast proteome down on a single [array] slide, so when you take a purified human protein... you can test it on this yeast proteome chip for potential interactions, which might actually give you a clue into what pathway the newly identified protein may actually fit in," says Arcot.

Arcot's caveat-filled endorsement has a familiar ring to it, as biochemists have long associated yeast-based systems with noisy assays. Unlike the standard yeast two-hybrid screen, though, an array of yeast proteins permits the experimenter to adjust the binding conditions, potentially reducing spurious interactions-or increasing them.

Whether they use the entire yeast proteome or a large set of human proteins, large-scale arrays are now limited to providing educated guesses about potential interactions. True high-throughput proteomics still runs up against the ugly side of biochemistry: protein purification. As each protein is different, purification remains as much art as science, forcing investigators to develop tailored protocols for isolating each protein. Until that laborious process is done for the millions of proteins in the human proteome, large-scale arrays will likely remain lowresolution tools.

With a clue from the large-scale arrays, though, researchers can develop moretailored approaches to characterize their novel proteins. Of course, there are arrays for that, too.

\section{Protein junction, what's your function?}

Once an experimenter has a short list of candidate binding partners, or a general idea of the pathway in which a protein participates, more specific functional arrays can clean up the picture. These are often arrays of purified proteins from distinct families, for example, an array of kinases, or cell cycle proteins, or all of the possible post-translational variants of a single protein. 


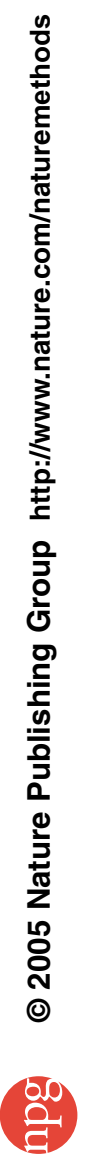


For these arrays to provide cleaner results than the initial proteome-scale tools, they need to be well-designed, so experimenters should study the commercial options carefully. As Hadjisavas explains, a successful functional array must meet several criteria: "Firstly, it will confer the correct conformation on the protein. The second thing is to have a technology which will [orient] the protein uniformly on the spot... as opposed to having a random orientation. The third criterion is to have the technology that can extend the protein away from the surface, so that it's not sterically hindered."

Those concerns become especially important when the goal is quantitation. "Unlike gene expression arrays, the dynamic range of proteins between samples can be almost ten orders of magnitude ... this is where knowing an absolute amount of the protein becomes even more important, because if you're just going on brightness, it could be fivefold more or tenfold more," says PerkinElmer's Arcot. If different protein spots on an array also have different degrees of denaturation or inactivation, even rigorous measurements of binding events become meaningless.

Conversely, a bit of homework can produce impressive results. Whether by studying and carefully choosing the right commercial products, or building a system from scratch, functional arrays can provide important new insights into biochemical pathways. MacBeath, for example, has developed his own strategy for probing the interactions of signaling proteins. He and his colleagues incubate custom arrays with eight different concentrations of each purified protein they wish to test, generating an apparent dissociation constant for each interaction. By doing the assays on arrays, they can construct complete networks of interactions very quickly.

"We're now able to take slices through that network at different affinity thresholds... and say what are only the highaffinity interactions, and then start adding in the lower and lower affinity interactions and see how these receptors change as you increase their level of interaction," says MacBeath.

Biochemists who want definitive binding curves have long lusted after Biacore's line of products, and the company is now making forays into the world of highthroughput array processing. Besides detailed binding data, their systems also offer the benefit of label-free readouts, obviating the need to attach a potentially troublesome fluorescent or enzymelinked tag to either protein involved in an interaction.

At first glance, the Biacore systems may seem like low-throughput technologies, as most only use arrays with one to four different proteins attached. The arrays, however, can be washed and reused repeatedly.

"You go in with your analyte over the surface, you detect the interaction, then you go in with some cleaning solution... and then you go in with a new analyte, and you can do that in an automatic fashion," says Stefan Lofas, vice president and chief scientific officer of Biacore. 


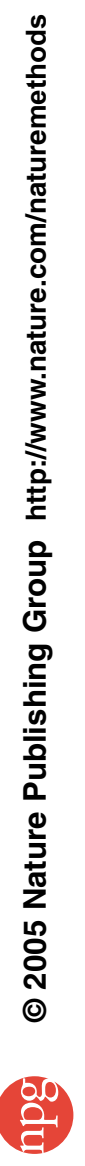


The company's T100 system, for example, can pull protein samples from a 384well plate and feed them automatically over a four-spot array, providing binding curves for all 384 samples against all four targets in 24 hours. The newer A100 system can do the same thing with a twentyspot array, yielding thousands of binding curves per day.

\section{Bits and pieces}

Not every functional array requires rigorous quantitation. Indeed, one of the most popular uses for functional arrays is searching for specific domains that interact with a protein of interest, an assay in which a simple yes or no answer is often enough.

Prefabricated domain arrays typically focus on popular, well-characterized domains. The $\mathrm{SH} 2$ and $\mathrm{SH} 3$ domains involved in kinase signaling pathways are especially popular, and several companies now offer arrays carrying those and related domains.
Epitope-mapping arrays are also handy, especially for those who want to identify the binding domains of antibodies. Like DNA arrays, these divide the target into short segments, usually ten to fifteen amino acids long, and tile it along the surface. The serially sectioned target provides a fast, direct readout showing which portions bind to the putative partner protein. Although the result must be confirmed with recombinant proteins in a more biologically relevant context, knowing where the interaction is likely to occur could shave months off a project.

Peptide arrays are also useful for identifying the antigenic portions of a protein, and many immunologists are now using them to determine which portions of an antigen are processed and presented on T-cell and MHC receptors.

Most peptide arrays are necessarily custom-built, as they focus on individual proteins, but that does not mean researchers have to build them from scratch. To fill the growing demand for these tools, many array makers now offer custom peptide services; send them an amino acid sequence, and they ship it back neatly segmented. Cambridge Peptides, New England Peptide, and Sigma, among others, now cater to the peptide array market.

Working with proteins will probably never be as easy as working with DNA or RNA, but recent advances in protein and peptide array design are providing some much-needed relief. Instead of quantifying and characterizing proteins one at a time, savvy experimenters can speed their work by running many of their assays in parallel, provided they keep the fundamental limits of those assays in mind. As both the quantity and quality of the results improve, researchers are now faced with the problem of processing all of the data. For the traditionally slow and meticulous science of biochemistry, it's a nice problem to have.

Alan Dove, Ph.D., is a freelance writer based in New York City (alanwdove@earthlink.net). 


\section{SUPPLIERS GUIDE: COMPANIES OFFERING ARRAY-BASED PRODUCTS AND SERVICES}

\section{Company}

Advalytix

Affibody

Affymetrix

Agilent

Applied Biosystems

Applied Precision

ARRM

Beckman Coulter

Becton Dickinson

Biacore

BioGenex

Bio-Rad

BMG Lab Technologies

Cambridge Peptides

Cell Signaling Technology

Dualsystems Biotech

GE/Amersham Biosciences

Genetix

Genomic Solutions

Greiner Bio-One

Hypromatrix

Invitrogen/Protometrix

KBiosystems

Molecular Devices

MorphoSys/Antibodies by Design

Nalge Nunc International

NanoPlex Technologies

NextGen Sciences

New England Peptide

EMD Biosciences/ Novagen

Panomics

Pepscan Systems

Peptide Specialty Laboratories

PerkinElmer Life Sciences

Pierce

Proteome Systems

Qiagen

RoboDesign

Scienion

Sigma-Aldrich

Stratagene

SuperArray Bioscience

TeleChem International

Whatman/Schleicher \& Schuell Bioscience

Zeptosens

Zyomyx

\section{Supplier}

http:// www.advalytix. de

http:// www. affibody.com

http:// www. affymetrix.com

http:// www.agilent.com

http:// www.appliedbiosystems.com

http:// www.appliedprecision.com

http:// www.arrm.com.au

http:// www. beckmancoulter.com

http:// www.bd.com

http:// www. biacore.com

http:// www. biogenex.com

http:// www.bio-rad.com

http:// www.bmg-labtechnologies.com

http:// www.cambridgepeptides.com/

http:// www.cellsignal.com

http:// www. dualsystems.com

http:// www5.amershambiosciences.com

http:// www.genetix.co.uk

http:// www. genomicsolutions.com

http:// www. greinerbioone.com

http:// www.hypromatrix.com

http:// www. invitrogen.com

http:// www. kbiosystems.com

http:// www. moleculardevices.com

http:// www. antibodyservices.com

http:// www. nuncbrand.com

http:// www. nanoplextech.com

http:// www. nextgensciences.com

http:// www. newenglandpeptide.com

http:// www. emdbiosciences.com

http:// www. panomics.com

http:// www. pepscan.nl

http:// www. peptid. de

http:// las. perkinelmer.com

http:// www. piercenet.com

http:// www. proteomesystems.com

http:// www. qiagen.com

http:// www. robodesign.com

http:// www.scienion.de

http:// www. sigmaaldrich.com

http:// www. stratagene.com

http:// www. superarray.com/ home.php

http:// www. arrayit.com

http:// www. schleicher-schuell.com/ bioscience

http:// www.zeptosens.com

http:// www.zyomyx.com 